\title{
Exocytosis provides the membrane for protrusion, at least in migrating fibroblasts
}

\section{Mark S. Bretscher}

Robert Kay and colleagues (Changing directions in the study of chemotaxis. Nature Rev. Mol. Cell Biol. 9, 455-463 (2008)) $)^{1}$ cite the popular view that actin polymerization drives the forward protrusion of moving cells and suggests that "membrane flow has received little experimental support". This statement disregards the following observations, based on fibroblasts, which suggest that exocytosis provides the surface for forward protrusion.

Endocytosis, which is driven by clathrincoated pits, occurs randomly and internalizes $\sim 2 \%$ of the cell surface per min. In stationary cells, exocytosis occurs randomly on the cell surface whereas, in migrating cells, exocytosis occurs at the leading edge. This main difference between stationary and migrating cells was discovered in spreading cells ${ }^{2,3}$ and was later extended to migrating fibroblasts ${ }^{4}$.

Given the rate of endocytosis, the rate at which exocytosed membrane could extend the front of a fibroblast is $\sim 1 \mu \mathrm{m}$ per min. Indeed, this is the speed with which these cells extend forwards, which suggests that the surface required for protrusion is provided by the endocytic cycle in fibroblasts. A more subtle point: as some fibroblasts move very slowly, the proportion of exocytosing membrane delivered to the front of the cell compared with the membrane that is delivered randomly might vary. If so, this would provide cells with a molecular clutch for varying their speeds.

'Membrane flow' refers to the consequences of this polarized endocytic cycle - that a flow, primarily of lipid molecules, occurs in the plasma membrane of the cell from the cell front towards the rear of the cell. Circulating receptors (such as transferrin receptors) become kinetically trapped towards the front of the cell ${ }^{2,3}$; before they can diffuse to the rear of the cell, they are recaptured by endocytosis and returned to the front. Non-circulating proteins should be swept backwards ${ }^{5}$, although this might be difficult to observe because individual molecules diffuse too quickly. However, such 'reverse' gradients have recently been found in polarized yeast cells ${ }^{6}$. The rearward movement of slowly diffusing complexes, such as particles that are attached to the cell surface $^{7}$ or cross-linked proteins $s^{8,9}$, are easier to observe. Besides providing an extension of surface at the front of the cell, exocytosis could also provide a fresh supply of feet for the cell to attach to the substratum ${ }^{10}$; this idea is supported by data in neutrophils ${ }^{11}$ and other cells.

The notion that membrane circulation is the motor for movement in fibroblasts remains to be confirmed, as is the case for actin polymerization. Furthermore, the contribution of membrane flow to Dictyostelium discoideum migration also remains unclear. Wild-type amoebae rapidly internalize their surfaces (every 4-10 mins ${ }^{12}$ ), which is compatible with their speed of movement. Conditional mutations in either $\mathrm{N}$-ethylmaleimide-sensitive fusion protein (NSF) or Sec1 (required for intracellular transport and exocytosis, respectively) block cell migration ${ }^{13,14}$, which indicates that membrane transport is required. But do all cells that have 'amoeboid' movement actually move by similar processes? And is D. discoideum a good model for mammalian cells? It would clearly help to discover where, on the D. discoideum surface, both endocytosis and exocytosis occur. This knowledge could help resolve a paradox: persuasive evidence indicates that a rearward flow of membrane does not exist in amoebae $^{15}$, but these same cells cap surface particles or aggregates, which I would otherwise interpret as indicating a flow.

Mark S. Bretscher is at the MRC Laboratory of Molecular Biology, Hills Road, Cambridge CB2 OQH, UK. e-mail:msb@mrc-Imb.cam.ac.uk doi: 10.1038/nrm2419-c3
Kay, R. R., Langridge, P., Traynor, D. \& Hoeller, O. Changing directions in the study of chemotaxis. Nature Rev. Mol. Cell Biol. 9, 455-463 (2008).

2. Bretscher, M. S. \& Thomson, J. N. Distribution of ferritin receptors and coated pits on giant HeLa cells. EMBO J. 2, 599-603 (1983).

3. Bretscher, M. S. Distribution of receptors for transferrin and low density lipoprotein on the surface of giant HeLa cells. Proc. Natl Acad. Sci. USA 80 454-458 (1983).

4. Hopkins, C. R., Gibson, A., Shipman, M. Strickland, D. K. \& Trowbridge, I. S. In migrating fibroblasts, recycling receptors are concentrated in narrow tubules in the pericentriolar area, and then routed to the plasma membrane of the leading lamella. J. Cell Biol. 125, 1265-1274 (1994).

5. Bretscher, M. S. \& Aguado-Velasco, C. Membrane traffic during cell locomotion. Curr. Opin. Cell Biol. 10 , 537-541 (1998)

6. Valdez-Taubas, J. \& Pelham, H. R. Slow diffusion of proteins in the yeast plasma membrane allows polarity to be maintained by endocytic cycling. Curr. Biol. 13, 1636-1640 (2003).

7. Abercrombie, M., Heaysman, J. E. M. \& Pegrum, S. M. The locomotion of fibroblasts in culture III. Movement of particles on the dorsal surface of the leading lamella. Exp. Cell Res. 62, 389-398 (1970).

8. Taylor, R. B., Duffus, W. P. H., Raff, M. C. \& de Petris, S. Redistribution and pinocytosis of lymphocyte surface immunoglobulin molecules induced by an anti-immunoglobulin antibody. Nature New Biol. 233, 225-229 (1971).

9. Bretscher, M. S. Directed lipid flow in cell membranes. Nature 260, 21-23 (1976).

10. Bretscher, M. S. Endocytosis: relation to capping and cell locomotion. Science 224, 681-686 (1984).

11. Lawson, M. A. \& Maxfield, F. R. $\mathrm{Ca}^{2+}$ - and calcineurindependent recycling of an integrin to the front of migrating neutrophils. Nature 377, 75-79 (1995).

12. Aguado-Velasco, C. $\&$ Bretscher, M. S. Circulation of the plasma membrane in Dictyostelium. Mol. Biol. Cell 10, 4419-4427 (1999).

13. Thompson, C. R. \& Bretscher, M. S. Cell polarity and locomotion, as well as endocytosis, depend on NSF. Development 129, 4185-4192 (2002).

14. Bretscher, M. S. \& Clotworthy, M. Using single loxP sites to enhance homologous recombination: ts mutants in Sec 1 of Dictyostelium discoideum. PLoS ONE 2, e724 (2007).

15. Traynor, D. $\&$ Kay, R. R. Possible roles of the endocytic cycle in cell motility. J. Cell Sci. 120, 2318-2327 (2007). 


\section{CORRESPONDENCE}

\section{ONLINE CORRESPONDENCE $\bigotimes$}

Nature Reviews Molecular Cell Biology publishes items of correspondence online. Such contributions are published at the discretion of the Editors and can be subject to peer review. Correspondence should be no longer than 500 words with up to 15 references and should represent a scholarly attempt to comment on a specific Review or Perspective article that has been published in the journal. To view correspondence, please go to our homepage at: http://www. nature.com/nrm and follow the link from the current table of contents.

The following correspondence has recently been published:

Exocytosis provides the membrane for protrusion, at least in migrating fibroblasts Mark S. Bretscher

This correspondence relates to the article:

Changing directions in the study of chemotaxis

Robert R. Kay, Paul Langridge, David Traynor and Oliver Hoeller

Nature Reviews Molecular cell Biology 9, 455-463 (2008) 